\title{
USP Dissolution Calibrators: Re-examination and Appraisal
}

\author{
Tom Foster ${ }^{1}$ and Will Brown ${ }^{2}$ \\ ${ }^{1}$ Chair of the USP Expert Committee on Biopharmaceutics \\ ${ }^{2}$ Corresponding author, USP staff liaison, USP, 12601 Twinbrook Parkway, Rockville, MD
}

email:web@usp.org

Note-The following represents the opinion of the chair and the staff liaison of the USP Expert Committee on Biopharmaceutics.

A s part of an oral drug product specification, dissolution testing is exceptional in that it assesses performance from a number of perspectives. This type of testing provides the formulator a range of indications from the summative quality of their product manufacturing processes to a surrogate assessment of potential in-vivo performance during the product's lifetime. Calibration of dissolution apparatuses is simply a demonstration of suitability for use in that test and, in concept, is similar to system suitability determinations for other analytical procedures. Dissolution performance is measured by the rate of appearance of the drug in solution and many other variables are at work to produce an observed dissolution rate. In the dissolution test the USP apparatus is merely the operational equipment source of the sample. The use of a standard tablet in dissolution apparatus calibration allows verification that the sample produced is representative of what might be considered best practices. In providing standard calibrator tablets, the USP accepts responsibility for reproducible performance of the calibrators and for setting appropriate criteria for calibration. The USP dissolution calibrator program is continually under surveillance and nurture by the Expert Committee on Biopharmaceutics, USP scientific liaison staff and a rich collection of volunteer stakeholders serving the dissolution testing community. The spirit and enthusiasm of this group will undoubtedly drive the scientific direction of the calibrator program in the years ahead.

\section{Overview of Dissolution Apparatus Calibration}

Dissolution testing is a powerful and discriminating technique for evaluating the performance similarity of successive lots of primarily solid oral dosage forms. Comparisons of performance characteristics of a given product or among various products require the test assembly to function in a standard and reproducible fashion. Dissolution failure is a leading cause of product recalls giving an added urgency for assurance that these test systems are suitably robust.

Dissolution testing is an umbrella term that takes into consideration both the sample preparation and its subsequent analysis. The analytical procedures used to generate the finished data reflect the high level of accuracy and precision associated with modern instrumental methods. However, ensuring that the variation seen in the analyzed drug concentration from multiple dissolution samples represents some intrinsic difference in the units tested and not the cumulative effect of small differences in the dissolution test environment presents a formidable challenge to the test platform. Variation in stirring speed, environmental vibration, and temperature are examples of perturbations occurring during testing that can affect the observed dissolution rate. Similarly, deviations in the vessel dimensions have been suggested as sources of variability in results (1).

The long standing approach to dissolution apparatus suitability by the USP has been to combine a sufficiently detailed description of the compendial testing apparatuses with a demonstration of suitable performance using reference standard materials, the dissolution calibrators. The process by which a test apparatus is determined to meet the compendial dimensional and operational specifications has been termed mechanical calibration, while the use of reference standard tablets is given the designation chemical calibration.

Chemical calibration allows a final and summative confirmation of the suitable operation of the integrated dissolution assembly, beyond the evaluation of its separate component attributes (e.g., stirring element dimensions, control of rotation speed, dissolution medium volume). It challenges the test assembly to perform its designed function as a sample preparation system. We believe that neither mechanical nor chemical calibration alone is sufficient to adequately furnish a robust demonstration of system suitability.

Advocates of the purely mechanical approach to calibration recommend that this should not be done until a concomitant tightening in the dimensional and operational tolerances given in the Dissolution General Chapter $<711>$ occurs (i.e., reduce tolerance on rotational speed from $\pm 4 \%$ to $\pm 2 \mathrm{rpm}$ ). However, we believe such suggested reduced tolerances are unfounded. Unless the current tolerances have been evaluated and found in need of adjustment and the proposed ideal tolerances are found to reduce the variability in results, no basis for the change is warranted or can be given. Regardless, any such study of the robustness of the dissolution as a sample preparation procedure will require a standard material. It is doubtful that any source of such a standard material other than the USP calibrators will be found. We therefore recommend that until such a study can be completed any attempt to set new tolerances is without merit.

\section{Calibrator Ranges}

USP conducts collaborative studies to determine expected values when a new lot of calibrators is tested under controlled conditions by qualified laboratories. Typically, between 10 and 40 laboratories from all over the world agree to participate. For each combination of Apparatus and 
speed, the data is subjected to a nested random effects model statistical method and the variance components from inter-laboratory, intra-laboratory, and inter-tablet sources are estimated. The ranges are determined as an interval around the mean of the data by adding or subtracting the product of a standard deviation term (incorporating the several sources of variance) and a constant representing the probability desired. Typically, a small fraction of the submitted results will fall outside of the ranges drawn.

Currently, individual data values obtained in a calibration test must fall within the range determined as described above. Acceptable results may fall anywhere within the range. The statistical methods used incorporate several sources of error in estimating the range of acceptable values. Two sources of variation of results, inter-tablet intrinsic performance variability and intra-laboratory variability, are of interest. The intrinsic performance from tablet to tablet within a production lot of calibrator tablets presents an underlying source of variability in results. An argument has been raised that the variability in intrinsic performance of the USP calibrator tablets is so great that it exceeds the variability of performance of modern test dissolution assemblies. Vessel-to-vessel variability of results, which includes tablet performance, is a significant source contribution to the magnitude of the calibrator ranges. Clearly, a standard reference material used to estimate the performance of a dissolution test assembly must exhibit an intrinsic performance with exceedingly low variability across the lot in distribution. The variability of inter-laboratory values brings an effect of similar magnitude to the width of the calibrator ranges. Incorporating the effect of inter-laboratory variability in the ranges without restricting the precision allowed in data from all vessels in one assembly reduces the value of chemical calibration. A lack of precision in calibration results is an indicator of problems in the operation of the assembly and should be used in the system suitability determination.

\section{Current USP Initiatives Salicylic Acid Tablets.}

This non-disintegrating calibrator tablet has suffered "image problems" over its history. Fused and chipped tablets may be found in bottles from the current lot. To correct this problem, USP staff has worked with a contract manufacturer to blister pack individual tablets in succeeding lots of tablets and thereby eliminate the occurrence of fused tablets. We anticipate that improved manufacturing and packaging procedures will dramatically reduce the number of flawed tablets

Salicylic acid calibrator tablets have also been criticized for alleged insensitivity to perturbational variables related to Apparatuses 1 and 2. Evaluation of studies examining these criticisms has currently not warranted a recommenda- tion to remove the salicylic acid calibrator. Further study is necessary and this is under consideration. Initial stages of the evaluation of the calibrator for its use in the calibration of Apparatus 4, the flow-through cell, are underway by USP.

\section{Prednisone Tablets.}

A focus of the USP Project Team on Dissolution Calibrators has been the concept of developing an 'ideal calibrator'. The current reference standard for a disintegrating calibrator is the 10-mg prednisone tablet. This tablet represents a modification of the NCDA \#2 tablets (see sidebar for discussion of calibrator tablet terminology) that have been shown to be sensitive to several operational variables by the Division of Pharmaceutical Analysis of the FDA $(2,3,4,5,6)$. The Project Team has recommended that USP attempt to reproduce a 50-mg prednisone tablet that was distributed by USP as its disintegrating calibrator until 1999. In an effort to determine if NCDA \#2, the current 10-mg USP reference standard, or the reproduced 50-mg prednisone tablet, is of value as an approximately ideal calibrator, the USP has committed to produce a demonstration batch for each of the three products and subject them to an abbreviated study of the response to perturbational variables for Apparatus 1 and 2 in a" head-to-head" challenge. The variables under study will be vessel symmetry, vibration, stirring element displacement (height), and wobble. The study is currently in the planning stage.

\section{Ideal Calibrator and "Side By Side" Evaluation.}

The USP dissolution test implies the concomitant system suitability demonstration. Results of dissolution testing must be obtained on a suitable apparatus. We encourage consideration of the following: in common with other analytical techniques such as HPLC, system suitability could be performed with a minimal number of additional samples required, with very little interruption of the workflow. Same-day or near-day calibration would increase the confidence in results and reduce the risk that quantities of data might be obtained on unsuitable apparatuses. In contrast, current dissolution apparatus calibration interrupts the workflow from a test assembly, requiring a different medium and volume, and often a different apparatus speed for each of two separate calibrator tablets. Most will agree that as currently designed, the calibration cannot be performed with the implied frequency, hence the present six-month interval between calibrations.

The current calibration design requires two separate conditions per apparatus. We believe that the ideal calibrator deployed "side by side" could give adequate control with only one test condition per apparatus. Ideally, the investment of time required should be reduced to the point where it could be performed, if not daily, then at least more frequently than current calibration testing. 
Along with the time spent in calibration, the laboratory risks a failing result. The time spent investigating and correcting the cause of failure as well as the possibility that a cause might not be found and the apparatus taken out of service all work against more frequent calibration testing. An ideal calibrator should be a diagnostic tool allowing a better estimate of the causes of failing results.

For a disintegrating dosage form to be used as a calibrator, the disintegration time must be uniform from tabletto-tablet. The duration of the test is set so that the dissolution process has not reached a plateau so as to give maximum sensitivity. The rate at which the individual tablets disintegrate has a great impact on the surface area exposed and thus on the variability of dissolution rate. The ideal calibrator, if a disintegrating dosage form, will be designed with this in mind.

\section{Comparator for In-Vitro Testing.}

Chemical calibration has the potential to go beyond the current demonstration of sameness of results among test apparatuses. The possibility exists for the use of calibration results to evaluate a rank order comparison of apparatuses that may be predictive of relationships that may be seen when those apparatuses are used in testing other marketed products. We have seen that, when compared, some dissolution test assemblies tend to give higher or lower results. In many countries, dissolution results are used as an estimate of bioequivalence between products. A standard reference product could serve to evaluate the comparative performance of individual test assemblies to aid in this effort and provide a basis for comparison of dissolution results obtained on different test assemblies.

\section{Conclusion}

The USP calibrator program is constantly being monitored and undoubtedly there will be changes in coming years to reflect the advancing science of drug product delivery, especially in the area of solid oral dosage forms. Changes to dissolution testing and calibration must be scientifically justified and meet the needs and views of the dissolution testing community. The continuing basis for the use of calibrators will be as it has been in the past, to demonstrate that a given dissolution assembly can function to produce a reproducible sample from the complex interaction of a moving solvent and drug product. The use of calibrators will grow as improvements are made in their utility as system suitability probes. Calibrators are your standard and USP is committed to work with the stakeholder community to enhance their value to the community.

\section{References}

1. DC Cox, Wells CE, Furman WB, Savage TS, King AC, Systematic Error Associated with Apparatus 2 of the USP Dissolution Test II: Effects of Deviations in Vessel Curvature from That of a Sphere, J. Pharm Sci, 71(4), 395-399; 1982

2. JF Hamilton, Moore TW, Kerner CM, Reproducibility of
Dissolution Test Results, PF 21(5), 1383-1386; 1995

3. TW Moore, Hamilton JF, Kerner CM, Dissolution Testing: Limitations of the USP Prednisone and Salicylic Acid Calibrator Tablets, PF 21(5), 1387-1396; 1995

4. TW Moore, Shangraw RF, Habib Y, Dissolution Calibrator Tablets: A Recommendation for New Calibrator Tablets to Replace Both Current USP Calibrator Tablets, PF 22(3), 2423-2428; 1996

5. TW Moore, Cox DC, Dissolution Testing: Collaborative Study of the In-house NCDA \#2 Dissolution Calibrator Tablets with USP Apparatus 2, PF 23(3), 4250-4255; 1997

6. TW Moore, Cox DC, Demarest DA, Dissolution Calibrator Tablets: A Scaled-up Lot of a New Calibrator Tablet Recommended to Replace Both Current USP Calibrator Tablets, PF 23(6), 5352-5363; 1997

7. Subcommittee on Dissolution Calibration, Pharmaceutical Research and Manufacturers of America (PhRMA), Dissolution Calibration: Recommendations for Reduced Chemical Testing and Enhanced Mechanical Calibration, PF 26(4), 1149-1166; 2000

\section{Calibrator Tablet Terminology}

50-mg Prednisone Tablet—Disintegrating tablet used in the calibration of Apparatus 1 (basket) and Apparatus 2 (paddle). Donated by an industrial source and Pharmaceutical Manufacturers of America member (PMA was the precursor organization to PhRMA). Began distribution in 1978 as Lot $F$ and continued in distribution until 1999. Lot $L$ was the final lot designation. Lot $L$ was used in the PhRMA mechanical calibration study (7).

NCDA Tablet \#1-A disintegrating 5-mg prednisone tablet used in early studies of the reproducibility of dissolution results by the FDA National Center for Drug Analysis in St. Louis, Missouri. Replaced by NCDA Tablet \#2 when supplies were exhausted. Note that the NCDA is now called the Division of Pharmaceutical Analysis (DPA).

NCDA Tablet \#2-A disintegrating 10-mg prednisone tablet used by the NCDA as an in-house dissolution calibrator. Its use was only recommended for the paddle apparatus. Numerous demonstrations of its sensitivity to perturbational variables were reported and it was used in the PhRMA mechanical calibration study $(2,3,4,5,6,7)$.

Current USP Prednisone Calibrator-A 10-mg disintegrating tablet developed by Ralph Shangraw at the University of Maryland with support of USP and NCDA. The tablet was intended to reproduce the performance characteristics of the NCDA Tablet \#2. First distributed in 1999 as USP Lot M. USP has manufactured three lots and is in the process of manufacturing the fourth (Lot P). 